\title{
UN MODELO DE ADMISIÓN A LA UNIVERSIDAd NACIONAL DE San Antonio Abad de Gusco: PROPUESTA TEÓRICA ALTERNATIVA
}

Jaime Pilco Loaiza

\section{INTRODUCCIÓN}

La Universidad Nacional de San Antonio Abad del Cusco, hace más de veinte años, selecciona a sus estudiantes con la consabida prueba objetiva que fácilmente permite cuantificar conocimientos y raciocinios y excluye, sin más, aspectos importantes de la capacidad y posibilidades de los jóvenes. No es que lo declarativo y conceptual carezca de importancia, sino que evaluar sólo estos aspectos en desmedro de otros, hace limitados los criterios para el acceso a sus claustros.

Con ese objetivo, se ha analizado y descrito tanto el modelo vigente de admisión como las instancias y situaciones con las que se encuentra relacionado, evaluando y caracterizando a los estudiantes admitidos con los criterios de selección del modelo; para después diseñar y elaborar los lineamientos de un modelo incluyente e integral de admisión, tomando en cuenta las condiciones básicas de aprendizaje, competencia y vocación de los postulantes.

Desde la perspectiva asumida, el hombre es un ser biosociopsicocultural. Esta es una concepción multidimensional, consecuentemente global; razón por la cual, la universidad no puede seccionar y valorar parcialmente las condiciones de los postulantes que quieren estudiar una profesión. Hay necesidad de tomar en cuenta el interés, la vocación, las habilidades, la información, el estado de salud, entre otros, como indicadores de las posibilidades reales que poseen los postulantes.

La presente propuesta es producto del estudio del sistema de acceso a la Universidad de San Antonio Abad, realizado como corolario de la Maestría sobre Investigación y Docencia en la Universidad Nacional Pedro Ruiz Gallo de Chiclayo. La alternativa que se propone parte de la concepción biosociopsicocultural del postulante con un perfil potencial real que supera las exigencias institucionales y comprende motivaciones, conocimientos y condiciones, concibe la correspondencia entre lo ofrecido por el joven y la demanda de la universidad, a partir del perfil ideal inicial propuesto por las Carreras Profesionales, el mismo que se constituye en base para la profesionalización especializada ulterior.

\section{IDENTIFIGACIÓN}

\section{A. Denominación}

Lineamientos de un modelo teórico incluyente e integral de admisión a la Universidad Nacional de San Antonio Abad del Cusco, a partir de las posibilidades reales de los postulantes y basados en las teorías sistémica, organizacional y dialéctica.

\section{B. Marco institucional}

Sistema Referente: Universidad Nacional de San Antonio Abad de Cusco.

Infra sistema: Componente Académico.

Subsistema: Componente Admisión.

\section{SínTESIS DE LA PROBLEMÁtica DE PARTIDA}

La Universidad Peruana, a pesar de algunas iniciativas innovadoras aisladas, no ha podido superar el problema de selección de postulantes. La Universidad de San Antonio Abad aún continúa aplicando una forma tradicional de ingreso, muy a pesar de que al interior del claustro y fuera de él, todo el mundo conoce sus limitaciones y parcialidad y aun así, se persiste con la misma práctica. Teóricamente, la llamada Prueba de Admisión, PdA, 
por prestarse a la cuantificación, es un rezago de la influencia que el conductismo ejerce todavía sobre la educación nacional. La $P d A$ se caracteriza por su densidad; utiliza la menos recomendada forma de prueba objetiva, la selección múltiple; privilegia el empleo de la memoria mecánica; impone presión antes y durante el examen, y lo que es hasta traumatizante, demanda un significativo tiempo de preparación a los jóvenes postulantes.

Respecto a las repercusiones del problema, igualmente, son preocupantes: insuficiencia de los contenidos conceptuales y declarativos de las $P d A$, trato desconsiderado de los postulantes por parte del aparato administrativo, movilización masiva de los estamentos institucionales, ingente presupuesto para su financiamiento, alteración de la actividad especialmente académica, actitud conformista frente a la situación, entre otras, con todas las consecuencias que esta situación acarrea.

\section{FundAMENTOS DEL MODELO}

\section{A. Fundamentos pSicológico-PEdagógicos}

Desde esta perspectiva, el problema de admisión es psicológico y pedagógico. Como hecho psicológico, provoca frustración, tensión, estrés, tanto entre los postulantes y familiares, como entre los profesores que participan en la elaboración de la $P d A$ como en su recepción. La propuesta, se basa en la concepción que la psicología tiene del hombre, como ser biopsicosocial, la misma que es enriquecida al considerar que el postulante es un ser biosociopsicocultural; reivindica la dimensión psíquico-comportamental, por constituir una de las más ricas posibilidades que los jóvenes poseen, pues pone en juego diferentes procesos, no sólo cognitivos, sino también volitivos y emotivos como actitudinales y de desempeños; privilegia la salud mental tanto como la salud orgánica, indispensables en cualquier proceso de formación; además, disminuye las tensiones y motiva confianza, en especial del postulante, al valorar gran parte de los aspectos de su perfil real.

En tanto hecho pedagógico, la propuesta responde a un proceso previo y complejo de educación/formación, generalmente escolarizado, de adquisiciones y procesamiento de información -habilidades, conocimientos, destrezas, actitudes, durante los cuales el aprendiz lee y analiza, reconoce patrones; sintetiza y resume; organiza, elabora y almacena la información- diversifica el requerimiento de competencias en base a las multidimensiones del postulante, biológica, social, psicológica y cultural. Incrementa la variedad de reactivos o formas de evaluación, a fin de reconocer la amplitud de sus posibilidades. La elaboración de la $P d A$ demanda tomar en cuenta: principios pedagógicos para la formulación de los ítems y cuestiones, dominio de conocimientos conceptuales y procedimentales, capacidad de graduación y sistematización de la información, entre otros.

\section{B. FundAMENTOS SOCIOLÓGICO-ANTROPOLÓGICOS}

El acceso a los estudios universitarios está inmerso en los campos de la sociología y la antropología. Se trata de una actividad en la que intervienen individuos, grupos y colectivos; en diferentes momentos y distintas acciones, de manera directa o indirecta; los postulantes que se constituyen en sujetos y centro del proceso; las familias como entorno inmediato, las instituciones de formación/preparación, la Universidad responsable de la selección. Igualmente, entidades sociales que participan de una dinámica coyuntural, unas veces y permanente otras, que comprometen modificaciones en las relaciones internas y externas, que demanda contar con presupuesto para garantizar el proceso y su culminación.

Por otro lado, las personas que participan en las interacciones: postulante-postulante, postulantes-administrativos, postulantes-docentes, universidad-comunidad, etc. toman en cuenta los derechos y deberes así como las responsabilidades de unos y otros, especialmentede los postulantes de diferentes procedencias. Estas relaciones no están exentas de la praxis valorativa y moral, la misma que regula y pondera las actuaciones y desempeños. Disminuye la prevalencia de ciertos reguladores de relaciones interpersonales al universalizar las manifestaciones y valores de la diversidad de procedencias culturales.

Por todas estas razones, se observa la necesidad de asumir el reto de encontrar propuestas alternativas basadas en principios y conocimientos tanto sociológico-antropológicos, como psicológico-pedagógicos.

\section{Objetivos}

a. Contribuir a la innovación de la modalidad de selección para el ingreso a la Universidad de San Antonio Abad.

b. Valorar las posibilidades multidimensionales, vocación y propósitos existenciales de los jóvenes postulantes. 
c. Abrir la Universidad a los jóvenes críticos, creativos y emprendedores.

\section{SECUENCIA ESTratégica GeNERAL SUGERIDA}

En términos metodológicos la propuesta que hacemos es consecuencia de un proceso previo, el mismo que comprende acciones diferentes realizadas en distintos momentos.

a. Observación y análisis inicial del estado de la situación, con la finalidad de identificar el objeto o problema, a fin de tener idea y conocimiento amplio del campo de estudio.

b. Delimitación del objeto y proposición provisional de objetivos.

c. Formulación de un plan general de investigación contributiva, precisando la metodología y los recursos necesarios, asignando el tiempo y costos aproximados, así como un cronograma de acciones.

d. Detección y recopilación de información referida al objeto de estudio.

e. Evaluación de las acciones realizadas y precisión de tareas específicas.

1. Construcción, prueba y administración de instrumentos de recolección de datos.

g. Procesamiento cuantitativo y cualitativo, y obtención de conclusiones.

h. Planeamiento general de formulación de propuesta teórica.

i. Diseño y descripción de lineamientos del modelo teórico, precisando estructuras y procesos, componentes, entrada, salida, objetivos, grupo de decisión, etc.

j. Evaluación y reajuste del modelo propuesto.

\section{CaraGterización de la PROPUeSTa} TEÓRICA

\section{A. Organización del SISTEMA GENERAL CNIVERSITARIO}

a. Localización del objeto de la propuesta

La estructura general comprende dos grandes componentes: macrosistema y microsistema; el primero constituido por el entorno de la institución que incluye los suprasistemasnacional, regional y local; los heterosistemasuniversidades públicas y privadas; los isosistemas-estudiantes y docentes, y el segundo y principal, abarca, el sistema referencial UNSAAC; los infrasistemasacadémico y administrativo; los subsistemas -admisión, formación y promoción, y los elementos-postulante, examen e ingresante. (Ver Gráfico 1)

b. Estructura del sistema general de la universidad En el estudio realizado, se ha asumido como sistema referencial, la totalidad institucional caracterizada por su denominación genérica de Universidad Nacional de San Antonio Abad de Cusco, cuya estructura comprende los siguientes componentes.

Sistema referencial. Para el caso del trabajo que se reporta, el sistema lo constituye la Universidad Nacional de San Antonio Abad del Cusco que, dicho sea de paso y en términos macro, guarda relaciones con heterosistemas que comprenden instituciones con características y propósitos semejantes, perteneciendo el nuestro al de las Universidades Públicas; isosistemas que

Gráfico No 1. Propuesı́, localización general del chijeto de la propuesta ${ }^{1}$.

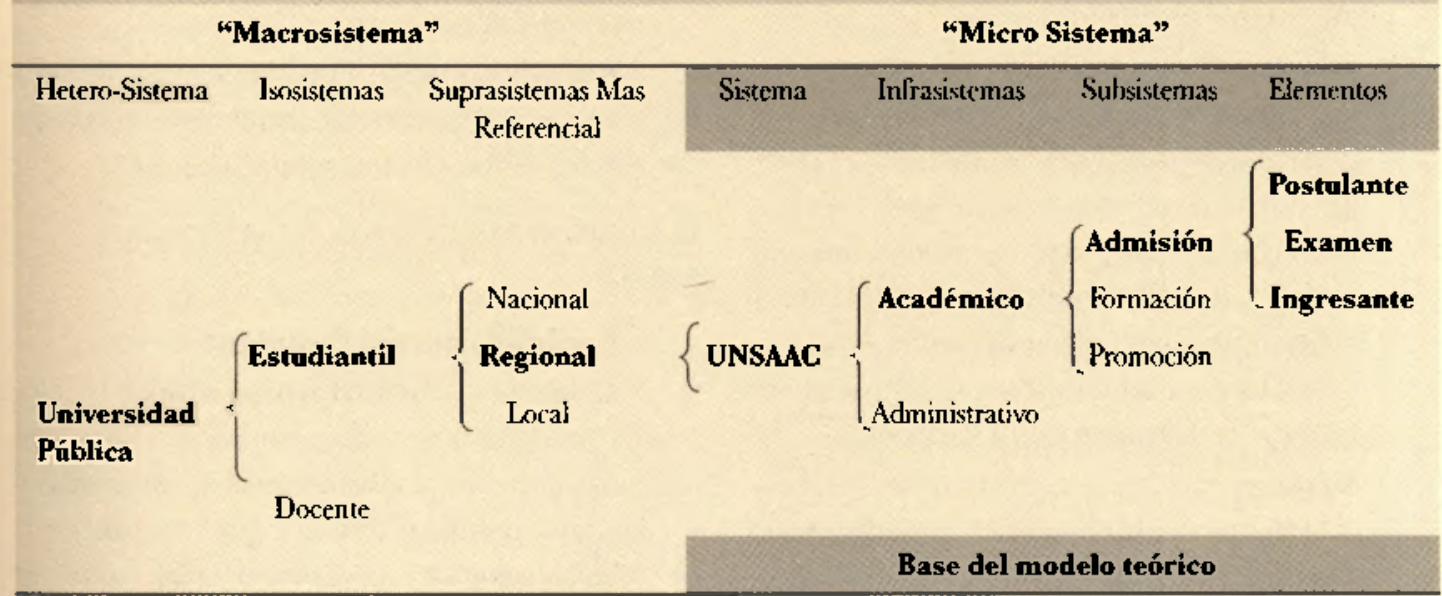

I. A parlir de Rodríguez. Valencia, Joaquin. 2003. Op.cit. pp. 39-44. 
involucran a docentes y estudiantes, razón de ser de las universidades; suprasistemas, contextos geopolíticos nacional, regional y local, los mismos que delimitan y precisan el sistema objeto de estudio.

Infra sistema académico. Constituido por el componente académico sugerido para el caso del sistema referencial, comprende tres subsistemas: admisión, formación y promoción.

Subsistema de admisión. Es la entrada al sistema donde el proceso mismo es el componente o evento académico-administrativo, el mismo que valora motivaciones, conocimientos y condiciones del postulante-ingresante, principal protagonista y componente académico por excelencia. Este subsistema fue el objeto de estudio y ahora objeto de la propuesta.

\section{Elementos del subsistema de admisión}

Postulante. Sujeto y objeto temporal del Subsistema de Admisión, por cuanto una vez superadas las evaluaciones a las que debe someterse, deviene en ingresante. Por esta razón, preferimos utilizar la denominación postulante-ingresante; además, se trata del informante por excelencia.

Ingresante. Situación nueva que adquiere el postulante al haber mostrado poseer las condiciones mínimas exigidas por la Carrera que desea estudiar.

Examen. Proceso de auscultación y valoración de las condiciones reales del postulante, a partir de un perfil ideal inicial requerido por la carrera profesional respectiva. La estructura del modelo integral de admisión centra su caracterización en este importante elemento.

Subsistema de formación. Constituye el escenario para la formación profesional y el desarrollo personal del estudiante; son parte de él los elementos académicos: estudios, investigación, proyección y producción, por un lado, docentes, metodología educativa y condiciones materiales, por otro. En cambio, los elementos administrativos son: recursos financieros, infraestructura y gestión.

Subsistema de promoción. Se trata del proceso culminante que promueve al egresado, previo "control de calidad", en el que se valora su calificación y competencia, garantía para un desempeño profesional satisfactorio en el mercado ocupacional.

B. MODELO INCLUSIVO INTEgRAL DE ADMISIÓN A LA Universidad Nacional de SaN ANtonio Abad de Cusco

Constituye la propuesta central operativa del presente trabajo; su estructura está conformada por:

a. Grupo de decisión

Conocido como Comisión de Admisión, encargada, en términos generales, de la organización, ejecución, seguimiento y evaluación del proceso en forma parcial y total.

b. Entrada

En la perspectiva estrictamente académica, comprende al postulante y su perfil real inicial o posibilidades con las que se presenta al examen de admisión.

c. Salida

La misma incluye el perfil ideal inicial propuesto por la carrera profesional y la situación nueva que adquiere el postulanteingresante.

d. Objetivos

Son propósitos que se espera lograr a mediano y largo plazo con los lineamientos del modelo de admisión propuesto.

e. Organización

Comprende la generación de contenidos a partir del perfil real de los postulantes, estructurado en base a las dimensiones, sub dimensiones e indicadores.

f. Evaluación

Denominado examen de admisión, utiliza reactivos específicos diversos como parte de los instrumentos generales para la captación de datos sobre los dominios, manejos y aprendizajes.

g. Retroalimentación que suponemos, durante la praxis, debe corregir, incrementar y mejorar el modelo conforme vaya aplicándose.

\section{Componentes generales que SLSTENTAN EL} MODELO

\section{Posibilidades del Postulante}

Criterio multidimensional. Se considera que el postulante sujeto de estudio es un ser biosociopsicocultural complejo y activo; poseedor de una estructura funcional biopsicológica, organismo $(\mathbf{O})$, que le permite adaptarse en los medios sociales y 
participar de su dinámica, respuesta $(\boldsymbol{R})$, a partir del desarrollo filo y ontogenético, modelado por la acción permanente de factores principalmente socioculturales, estímulos $(\boldsymbol{E})$, origen de su accionar. Se trata de una entidad con diferentes dimensiones, que refleja las muchas condiciones $y$ posibilidades del postulante. El gráfico trata de resumir este propósito.

Dimensión bioneural. Es consecuencia de los logros de un prolongado proceso filogenético, el mismo que ha garantizado el desarrollo ontogenético, constituido por un complejo de componentes que va desde las células hasta los grandes sistemas, cuyo funcionamiento unitario posibilita captar la realidad a través de los órganos sensoriales, para luego derivarlos a otros componentes de un desarrollo de mayor complejidad, encargados del más importante y admirable proceso $(\mathrm{O})$, gracias al cual participamos de las bondades que brinda el entorno $(\boldsymbol{E})$ y estamos permanentemente preocupados por transformarlo y cambiarlo.

En ese sentido, cualquier forma de valorar o calificar al ser humano (postulante/ estudiante) no puede dejar de comprender esta fundamental dimensión común, factor condicionante del desenvolvimiento $y$ desempeño de las personas.

Dimensiones social y cultural. Aquel desarrollo individual conseguido ha permitido un equipamiento con una estructura capaz de aprehender el mundo sociocultural y sus variantes, y aprender de él con el común propósito de recrearla. $\mathrm{El}$ postulante o estudiante, como cualquier otro, es producto social y el mayor hacedor de cultura; él construye la filosofia, la ciencia, el arte, la religión, la ética, las diferentes manifestaciones de los pueblos. Ese contexto social y cultural, es a su vez fuente de sus formas de pensar, sentir, decidir y actuar, por tanto, inagotable surtidor de saber $y$ conocimiento; y por otro lado, el entorno socio-cultural es receptor y destino de la diversidad de producción humana.

Este entorno como permanente origen principal de estímulos posee una dinámica significativa en la modelación psíquicocomportamental y como tal, en la orientación de las relaciones entre personas de los diferentes colectivos; suficiente motivo para preocuparse por considerarla en los criterios de acceso a los estudios superiores universitarios.

Dimensión psicológica. Es otro de los importantes componentes del postulante/ ingresante, aquello comprometido con su potencialidad psíquico-comportamental, capaz de permitir acomodarse a las permanentes exigencias de una realidad cada vez más dificil. Las formas de pensar, sentir, decidir y actuar delimitan la psicología de las personas; por tanto, comprenden los procesos cognitivo, emotivo y volitivo, por un lado, y comportamental por otro; los mismos que, en gran medida, son adquiridos y desarrollados a lo largo de las historia personal de cada uno. Se trata de recursos con los que no sólo el joven interactúa con el mundo, sino, se adapta a su transformación y participa de ella; consiguientemente, su acrecentamiento específico y especializado será objeto de la formación profesional, la misma que lo instrumentará para actuar en condiciones favorables frente a las exigencias del mundo real.

El perfil psicológico es consecuencia del desarrollo biopsicológico y la acción sociocultural sobre él, razón por la cual su modelación se constituye en objetivo de los estudios profesionales; hecho este que obliga a la universidad, reconocer y participar en su construcción y perfeccionamiento.

D. Carícter subdimensional (POSIBILIDAdes)

Las múltiples dimensiones del postulanteingresante permiten identificar sus reales condiciones, a partir de sub dimensiones concurrentes.

Salud fisica. El quehacer mental y comportamental demanda contar con un estado regular de salud orgánica, indispensable para prepararse y calificarse, consecuentemente participar de cualquier empresa formativa y productiva.

Destreza motriz. Aun pareciendo una exigencia escolar la posesión de motricidad fina, los jóvenes requieren para desenvolverse en diversos campos de la actividad profesional contar con instrumentación tan básica, por cuanto que siendo más intelectual el proceso de formación, éste demanda de dominios manuales coordinados y específicos. 
Resocialización. Entendida como el reforzamiento de la socialización, a través de diferentes estrategias, las mismas que permiten el reacomodo en los grupos en los que el joven se desenvuelve. Son importantes para evitar conflictos, competir lealmente, cooperar, motivar, obtener colaboración. Se debe contar con un mínimo de estos instrumentos; pero además, el sistema universitario tiene que prever, reconocer y ponderar los existentes e incrementarlos oportunamente, mediante mecanismos de autorregulación, integración y producción; desde luego, sabiéndolos planificar, monitorear y evaluar.

Conocimientos. Constituyen el bagaje de información acumulada a lo largo de la vida y referida básicamente a dos grupos de ellos: la cultura en general y los relacionados al campo específico de la carrera a la que se pretende acceder y luego ejercer. En todo caso, se considera importante evitar evaluar un margen amplio y basto de conocimientos, que sólo provocan frustraciones, desánimos y aislamientos.

Valores. Son condición del perfil más preciado en las personas, conformada por virtudes que la sociedad ha ido inculcando para regular las relaciones interpersonales e intergrupales y facilitar la convivencia humana. Se requiere un mínimo de valores para emprender la profesionalización y acometer el desempeño; entre muchos otros, la tolerancia y colaboración.

Salud mental. Es el indicador de estabilidad en las relaciones interpersonales de ausencia de perturbaciones psíquicas y comportamentales, hecho que permite orientar las acciones y los esfuerzos al logro de objetivos. Tanto la formación profesional como el desenvolvimiento profesional demandan de estas características; por tanto, el joven debe poseerlas y la Universidad está obligada a valorarlas y contribuir a su plasmación.

Aptitudes vocacionales. Las distintas ocupaciones laborales y profesionales requieren contar con ciertas habilidades y capacidades que faciliten y viabilicen la realización y cumplimiento de las acciones propias, motivo por el cual, hay necesidad de requerirlas al joven con el objeto de garantizar su profesionalización. En términos generales y para todos los casos, se debe estar motivado, interesado y contar con información sobre la carrera respectiva.

Aptitudes para los estudios superiores. Son las condiciones generales básicas e indispensables para el aprendizaje que el postulante/ ingresante debe poseer. En ese sentido, es importante el hábito de estudio; es decir, contar con prácticas cotidianas, como estudiar en un mismo lugar, a las mismas horas, de la misma manera; saber leer y resumir; conocer y utilizar organizadores de información; captar, procesar y utilizar información. Igualmente, conocer el estilo de aprendizaje personal, o sea de la forma cómo cada uno aprende mejor: (Solomon, 1999: 41)

$\begin{array}{ll}\text { Activo } & \text { Reflexivo } \\ \text { Sensorial } & \text { Intuitivo } \\ \text { Visual } & \text { Verbal } \\ \text { Secuencial } & \text { Global }\end{array}$

La universidad debe identificar esos hábitos y estilos, estimular e incrementarlos, pero además, evaluarlos.

Habilidades intelectivas. Son los requerimientos que el postulante/estudiante debe mostrar para la realización de un estudio efectivo y aprendizaje productivo; por ejemplo, la búsqueda, adquisición, conservación, recuperación y organización de información; inventiva y creatividad, análisis, toma de decisiones, comunicación, etc. Desde luego, no pueden dejarse de incluir aquellos procesos superiores como el pensamiento y lenguaje, las inteligencias, la memoria.

Actitudes. Entendidas como la disposición para actuar de una u otra forma. Se adquiere en el proceso de socialización que orienta la organización subjetiva del mundo, cuya modelación responde a las condiciones de vida y que es susceptible de ser modificada. La actitud es una forma de reacción que diferencia a las personas; por tanto, merece no sólo ser conocida, sino tomada en cuenta en el acceso a la Universidad y durante el proceso de formación profesional. Del mismo modo, es conveniente reconocer y alentar la actitud emprendedora de los postulantes/estudiantes. Consecuentemente, un sistema de admisión como el que se 


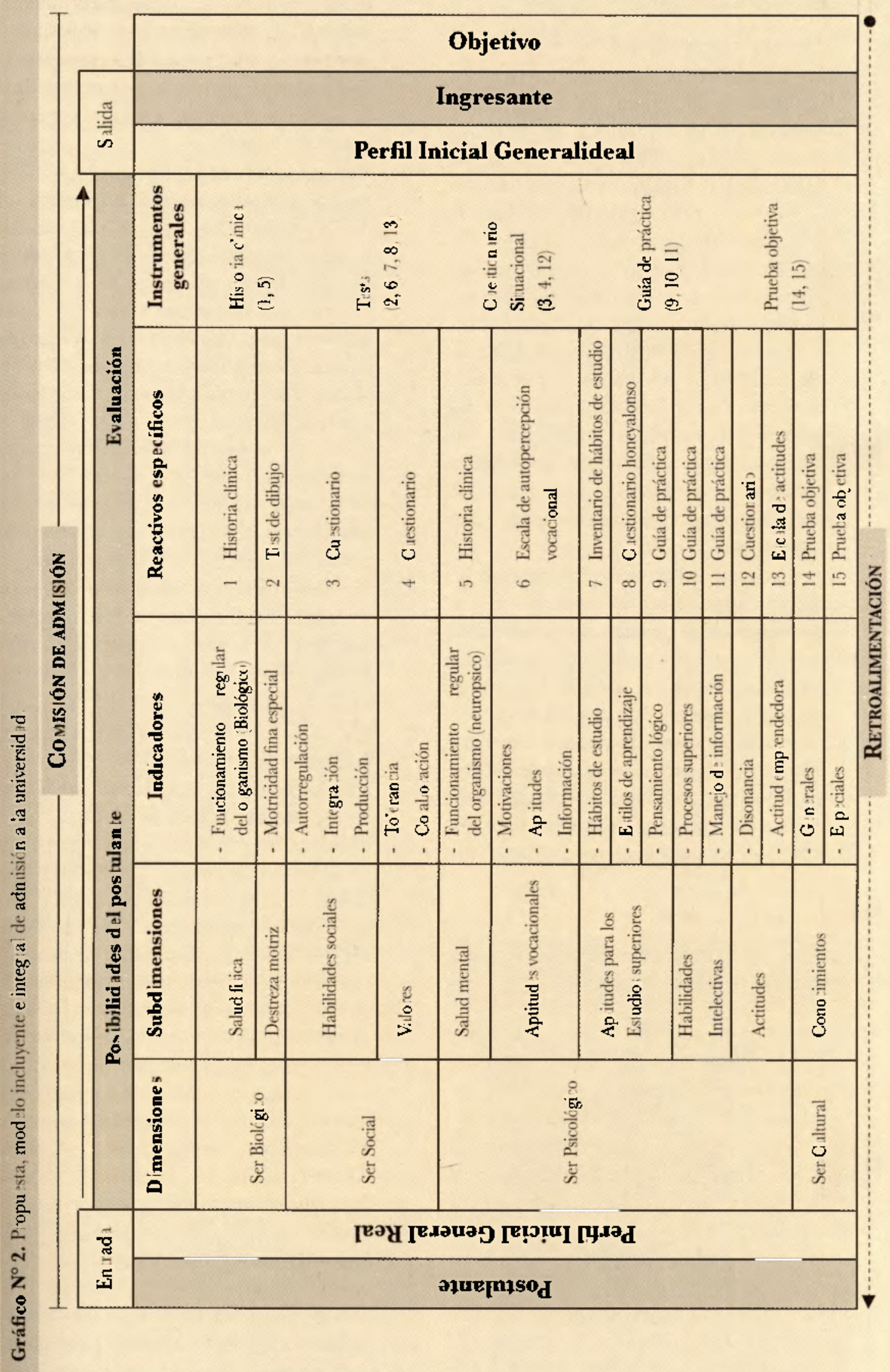


pretende, tiene que tomar en cuenta esta compleja multidimensionalidad del potencial de los jóvenes postulantes.

\section{Presencia multiteórica}

La universidad de estos días, en términos generales, acude a un común propósito de participar en la dinámica del desarrollo de los pueblos; la universidad pública de países como el Perú, aún continúa tratando de sobrevivir, preocupada por el futuro de los jóvenes de los sectores populares. La contribución de la universidad a los destinos del país, desde la perspectiva de la propuesta, académico, desde sus bases o entradas; en ese sentido, se considera prioritario, revisar la forma de admisión, al margen de los propósitos economicistas privilegiados. La admisión universitaria tiene implicancias sociales; son los hijos del pueblo los que demandan garantizar, en cierto modo, un futuro seguro.

Al margen de lo que se diga respecto de la universidad nacional, lo cierto es que sus usuarios son seleccionados, y rigurosamente, aunque no con los mejores criterios.La selección a la que se someten los jóvenes del pueblo pasa por competir con decenas o cientos de otros postulantes; por tanto, y esto ocurre en algunas carreras profesionales, son estudiantes con niveles considerables de calificación, con un perfil que se aproxima a lo óptimo; pero aun así son los menos. Lo

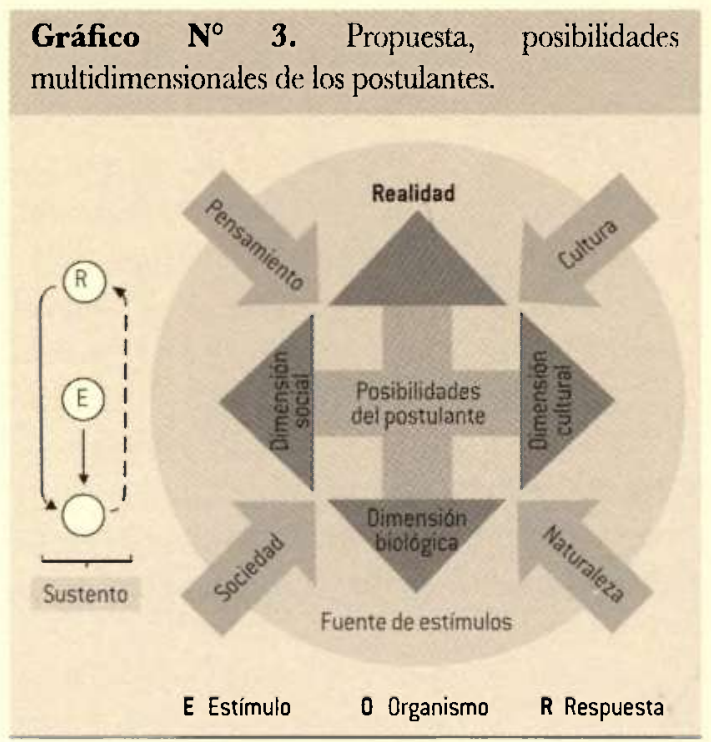

real sería que la mayoría de jóvenes tenga esa configuración; eso se pretende, que un mayor número de jóvenes con condiciones califique y acceda a la universidad. Para ese propósito existe la necesidad de modificar las reglas de juego, tal que se pondere la potencialidad que no aflora con los indicadores mnemotécnicos y reproductivos.

Para que la universidad estatal responda a las exigencias de estos tiempos, en el momento de la admisión hay que invertir más en la selección que estar preocupados por el recaudo. De ese modo, serán un mayor número quienes ingresen con un perfil suficiente para garantizar esa formación integral y competitiva. Se necesita cambiar los esquemas pecuniarios por otros de orden superior, los académicos; y qué mejor, a partir de las posibilidades y condiciones multidimensionales $y$ reales de los postulantes, que junto con los componentes teóricos han permitido la construcción del modelo incluyente e integral de admisión.

Por estos propósitos, la propuesta se orienta en sistemas de conocimientos provenientes de tres concurrencias científicas vigentes, las teorías sistémicas, de las organizaciones y la dialéctica.

Modelo sistémico. La Universidad, en este caso de San Antonio Abad, por la función que cumple, es un sistema cerradoabierto por cuanto tiene cierta autonomía, pero al mismo tiempo está relacionado con otros sistemas y el entorno; además, por su naturaleza, es un sistema social y artificial, en el decir de Bunge, instituido con un propósito socioeducativo que responde a la demanda del cultivo de las humanidades, la ciencia y la tecnología que requiere de la contribución de sus integrantes docentes, estudiantes, egresados y servidores administrativos. Se trata de una organización formal; esto es, un todo formado por diferentes componentes interrelacionados cuya acción está orientada a conseguir determinados objetivos, obedeciendo a un proceso planificado, sistemático y ordenado.

Como un todo organizado, la Universidad está constituida por dos grandes componentes: el académico y el administrativo, ambos interrelacionados, aunque no complementados plenamente. Aun existiendo deficiencias en las relaciones 
mutuas entre estos componentes, las actividades que ejecutan tienen un propósito institucional común.

Tanto el estudio como la propuesta no aborda, tampoco comprende, la totalidad del sistema universitario, únicamente el infra sistema académico y dentro de este, el referido al subsistema de admisión, en el que identificamos tres tipos de componentes: estructurales, funcionales y situacionales.

Los lineamientos del subsistema de entrada/ admisión comprende trestipos de componentes: temporales, funcionales y situacionales.

Componentes temporales. Delimitan los momentos del proceso en el que ocurren diferentes acciones específicas y que denominamos antes, durante y después.

Componentes funcionales. Comprende la entrada, el proceso y la salida (Rodríguez, 2003: 240); aluden a los componentes sistémicos donde la entrada corresponde a las acciones que se ejecutan y los recursos que se consideran previos al desenvolvimiento central o principal conocido como proceso. En él y de manera dinámica se desarrolla lo previsto y planeado en tiempos delimitados y conducentes al logro de determinados propósitos; conseguidos los objetivos en la salida, el sistema promueve la aplicación de los mismos por parte de los postulantes, en forma de rendimiento.

Componentes situacionales. Están referidos a la condición del postulante que adquiere en determinado momento el joven egresado de educación secundaria, el examen de admisión al que se somete y la calidad de ingresante que consigue una vez superadas suficientemente las exigencias de la prueba de admisión, dándole el estatus de estudiante universitario.

Modelo organizacional. En este modelo propuesto la admisión es una organización entendida como categoría sistémica que

Gráfico $\mathbf{N}^{\circ}$ 4. Propuesta, componentes temporal, funcional y situacional de la organización.

\begin{tabular}{l|cc:c} 
Componente & \multicolumn{3}{|c}{ Admisión } \\
\hline Temporal & Antes & Durante & Después \\
Funcional & Entrada & Proceso & Salida \\
Situacional & Postulación & Examen de admisión & Ingreso \\
\hline
\end{tabular}

alude al proceso previo a las ejecuciones y realizaciones, relacionando las cuales con la planificación, está orientada a lograr un fin mediante actividades y empleo de recursos materiales y humanos. La Universidad de San Antonio Abad cuenta con una Comisión Permanente de Admisión encargada de ordenar las acciones, definir la estructura y realización de actividades en las que intervienen profesores $y$ administrativos, así como el empleo de bienes materiales y presupuesto; por tanto, se trata de una organización formal, cuya constitución se basa en criterios legales y sociales, con objetivos, atribuciones y funciones claros; prevé el seguimiento, control y evaluación; además, obedece a un proceso planificado, sistemático y ordenado.

Las organizaciones como la Universidad comprenden partes y relaciones. En el caso que nos ocupa, las partes están delimitadas en el tiempo y relacionadas en la praxis. Las partes son módulos orgánicos de actividad, personas y recursos; en tanto las relaciones, son las interacciones multidireccionales entre los componentes y sub componentes. Todos los componentes temporales, funcionales y situacionales organizados conservan relaciones mutuas, por tanto constituyen un todo y como tal, su presencia y acción tiene un mismo propósito: la selección de jóvenes para su formación profesional.

Modelo dialéctico. Se trata de otra característica del sistema de admisión que se propone, por cuanto como hecho social se sustenta en los siguientes argumentos dialécticos.

Como sistema y organización constituye una unidad a pesar de la diversidad y contradicciones internas y externas, fundamentales y secundarias, antagónicas y no antagónicas existentes.

Participa de la transformación de la cantidad en calidad; es decir, concibe que lo alcanzado hasta ahora sea susceptible de ser superado, en su complejidad, magnitud, número de componentes, etc., aun teniendo características estables que la diferencian de otros sistemas.

Implica la correlación existente entre lo viejo y lo nuevo, por cuanto considera que como propuesta se basa y está relacionado con todo lo valioso y positivo del sistema precedente. 
El modelo del sistema de admisión en su plasmación resulta siendo la unidad de la teoría y la práctica, por ser resultado de la investigación realizada, para la cual se ha partido de la teoría confiable necesaria y de este modo haber descubierto las carencias y limitaciones existentes y a través de él mejorar los propósitos de la selección de postulantes (praxis) a la Universidad.

El modelo propuesto asume que como todo hecho o proceso, la admisión es dinámica y su tendencia al cambio implica transformación y desarrollo. Sus componentes y sub componentes están vinculados, existiendo dependencia y condicionalidad entre ellos.

\section{Lógica del infrasistema académico general}

La lógica académica general sugerida está conformada tanto por la estructura como por la dinámica propuesta respecto a la secuencia de acciones previstas para el acceso, profesionalización y promoción del joven.

La entrada al componente académico es el subsistema admisión, acceso que se plasma a través del examen de ingreso al que se somete el postulante con el propósito de que la institución valore su perfil real (motivaciones, conocimiento y condiciones) a partir del perfil ideal inicial o de entrada propuesto por las carreras profesionales. De este subsistema se ocupa el trabajo.

En el proceso se estructuran los lineamientos académicos de la formación profesional y desarrollo humano del estudiante, en base al perfil ideal inicial o de entrada y del perfil ideal al finalizar los estudios, los que deberán estar orientados en un proceso sistemático y gradual al logro de objetivos.

La salida comprende la situación de egresado que primero adquiere el estudiante al conseguir reunir las condiciones del perfil ideal final, que luego de su titulación lo convierte en profesional. Esta lógica no es nueva, es la que se halla en vigencia.

\section{Lógica del subsistema de admisión}

Comprende el objeto de estudio, propiamente dicho, que en la lógica general vendría a ser la entrada. Consideramos importantes tres componentes: El Perfil Real Inicial, el Examen de Admisión y el Perfil Ideal Inicial.
El perfil real inicial, el mismo que representa la concepción multidimensional del postulante, por el que se considera al sujeto de estudio como un ser biosociopsicocultural; son éstos los campos en los que hay que buscar las potencialidades, condiciones - posibilidades del futuro estudiante universitario; sólo así, la selección será coherente, integral, objetiva y pertinente; cualquier forma o modalidad que se emplee en la selección del postulante, debe considerar este complejo multidimensional. En ese sentido, el examen de admisión tiene que basarse en el perfil real inicial del postulante, y no en aquel exclusivamente escolar/académico pues sólo privilegia los conocimientos; esa ausencia hace del modelo vigente de acceso a la Universidad, parcial, limitado e insuficiente, con las consecuencias

Gráfico $\mathbf{N}^{\circ}$ 5. Propuesta, lógica académica del subsistema de admisión.

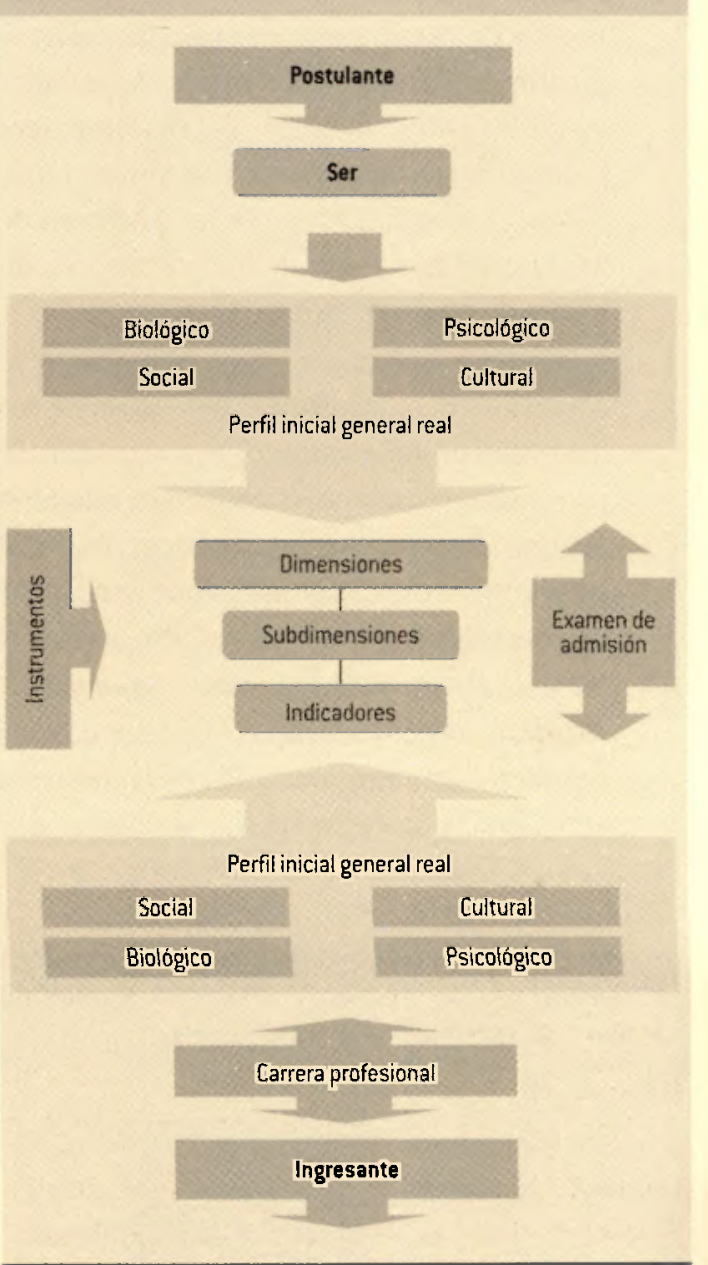


observables. Por este motivo, la propuesta considera diferentes instrumentos que valoran ese perfil, a partir de dimensiones, indicadores e índices.

Para completar el modelo propuesto se asume como indispensable que la Universidad, a través de sus Carreras Profesionales, exija a los postulantes satisfacer las exigencias de un Perfil Ideal Inicial o de entrada que comprenda las dimensiones a las aludidas. Sólo así se podrá evaluar en términos integrales y objetivos los requerimientos para garantizar la formación profesional.

\section{F. INSTRUMENTOS DE EVALUACIÓN}

\section{Descripción general de instrumentos}

Historia clinica. Examen médico y psicológico general para detectar el estado de salud fisica y mental a través de la evaluación del funcionamiento regular biológico y neuropsicológico; el mismo que permitiría garantizar la presencia de condiciones mínimas necesarias por parte de los postulantes para participar de un proceso complejo y riguroso de formación personal y profesional.

\section{Test}

Test de dibujo. Sobre trazos a mano alzada, para valorar la destreza motriz fina en la escritura, construcción de organizadores y elaboración de gráficos en general.

Escala de autopercepción vocacional. Reactivo sobre cuestiones relacionadas con las aptitudes, intereses, información e inclinaciones ocupacionales, a fin de identificar el arsenal de condiciones personales, facilitadores para la plasmación de la formación profesional.

Inventario de hábitos de estudio. Previstos con el propósito de reconocer la disposición para el estudio, la distribución y administración del tiempo; la organización de acciones propias de las diferentes tareas que demanda estudiar asignaturas teóricas y prácticas; la perseverancia, seriedad y responsabilidad con la que se asume formarse. Se trata de condiciones básicas para emprender estudios superiores.

Cuestionario de estilos de aprendizaje HoneyAlonso. Referido a las formas de aprendizaje individual, gracias al cual se puede identificar la modalidad sensorial, de conceptualización, categorización y reflexión.
Escala de actitudes. Para conocer la iniciativa en el desempeño, las posibilidades emprendedoras, el espíritu innovador y el liderazgo, necesarias en la empresa académica y laboral.

\section{Cuestionario situacional 10\%}

Útil en el descubrimiento de las posibilidades de organización cognitiva y comportamental que facilite el ajuste a patrones culturales diferentes, para de este modo viabilizar la integración y el consecuente incremento de las posibilidades personal y grupal.

Igualmente, el cuestionario hará accesible conocer la actuación compatible con las exigencias de la formación y desempeño profesional productivo, a través de la tolerancia y la colaboración en el trabajo grupal tanto académico como ocupacional. También identificar la posesión de un pensamiento y comportamiento coherentes para reconocer la actuación confiable en diferentes situaciones y entornos de desenvolvimiento.

\section{Guía práctica 10\%}

El instrumento aborda tres ámbitos sumamente importantes: pensamiento lógico, procesos psíquicos superiores y manejo de información. En ese sentido posibilita apreciar:

a. El rigor del pensamiento en situaciones comunes y científicas para valorar las capacidades de razonamiento que permitan argumentar y concluir.

b. La manifestación regular de procesos complejos superiores de atención, inteligencia y creatividad principalmente, indispensables en la formación y desempeño profesional.

c. La adquisición, procesamiento y aplicación de información para indagar los mecanismos de captación, organización, elaboración, conservación y aplicación de aprendizajes.

\section{Prueba objetiva 40\%}

Permite auscultar el dominio y reflexión acerca del conocimiento general y científico; es decir, información sobre asuntos sociales, culturales, artísticos diversos, como también niveles de información confiable vigentes en el campo de las humanidades, las ciencias naturales, ciencias sociales y tecnologías, priorizando aquellos conocimientos pertinentes a la carrera profesional. 
Gráfico $\mathbf{N}^{\circ}$ 6. Propuesta, orden de aplicación de instrumentos.

Secuencia

Criterios Primero Segundo Tercero

Contenidos Condiciones Información Salud

de la

evaluación

\begin{tabular}{llcc}
\hline Instrumentos & - Test & - Cuestionario & - Historia \\
generales de & - Guía de & situacional & clínica \\
evaluación & práctica & - Prueba objetiva & \\
\hline
\end{tabular}

G. Aplicación de instrumentos

Por la riqueza de aspectos que deben para facilitar los aprendizajes, viabilizar una formación suficiente y garantizar el desempeño profesional eficiente del joven, consideramos que en un solo evento resultaría poco recomendable evaluar, y que lo más atinado sería hacerlo en tres momentos deferentes.

Partimos de asumir semejante proceso de selección como la más importante de las responsabilidades que una institución como la universidad tiene respecto a la comunidad, por cuanto será la encargada de formar a los futuros promotores, conductores y transformadores del desarrollo de los pueblos, tarea trascendente. En ese sentido, los siguientes serían los tres momentos de la evaluación.

\section{BibLIOGRAFÍA}

ÁLVAREZ SAYAS, Carlos M. y SIERRA LOMBARDÍA, Virginia $M$.

2003. La Universidad: su gestión y su evaluación. Cochabamba. Bolivia, Kipus.

\section{ASAMBLEA NACIONAL DE RECTORES}

2008. Resumen estadístico universitario 2006. Lima, Dirección General de Planificación Universitaria y Dirección de Estadística e Informática.

CAPELLA RIERA, Jorge y otros

2003. Estilos de aprendizaje. Lima. PUCP

CARTER, Carol y LYMAN KRAVITS, Sarah

1997. Orientación vocacional: cómo alcanzar tus metas. México, Prentice Hall Hispanoamericana.

CÁZARES, Fidel y LóPEZ, José Luis

2006. Pensamiento crítico. México D. F., Pearson y Prentice Hall. CLAVIJO GAMERO, Rocío

2007. Pruebas psicotécnicas. México D. F., Limusa y Noriega Editores.

DE BONO, Edward

2000. El Pensamiento lateral, manual de creatividad. Buenos Aires, Paidós Plural.

DELORS, Jacques

1996. Informe a la UNESCO de la Comisión Internacional sobre la Educación para el siglo XXI. Madrid, Santillana.

\section{FORO EDUCATIVO y Consorcio de Universidades}

2001. La universidadi que el Peri necesita: encuentros y seminarios 1999-2000. Lima.

GARCÍA ROCA, Joaquín y MONDAZA CANAL, Guillermo

2002. Jóvenes, universidad y compromiso social: una experiencia de inserción Comunitaria. Madrid, Nancea.

\section{GONZALES, María Teresa}

2003. Organización y gestión de centros escolares: dimensiones y proceso. Madrid, Pearson - Prentice Hall.

HODGE, B. J.; Anthoni, William P. y GALES, Lawrence $M$.

2003. Teoria de la organización: un enfoque estratégico. Madrid, Pearson - Prentice Hall.

HUERTA AMEzolA, J. Jesús y PÉRez GARCÍA, Irma Susana

2002. Infuencia de algunos modelos universitarios en la Universidad de Guadalajara. México D. F., UDLAL.

\section{KURSÁNOV, G.}

1975. Materialismo dialéctico. Buenos Aires, CARTAGO.

\section{MINISTERIO DE EDUCACIÓN DEL PERÚ}

2005. Diseño curricular nacional de educación básica regular. Lima, DINEIP - DINESST.

2007. Guia de diversificación curricular. Lima, DNEBR - DES.

\section{PISCOYA HERMOZA, Luis}

2005. Calidad de las pruebas de admisión en la Universidadi Peruana. Lima, Instituto Internacional para la Educación Superior en América Latina y el Caribe y Asamblea Nacional de Rectores.

\section{RAMOS, Gerardo.}

1999. La Universidad Peruana en el siglo XXI Lima, Universidad Ricardo Palma.

RIQUELME, Graciela C.; BRUSILOVSKY, Silvia y otros

1998. Politicas y sistemas de formación. Buenos Aires, Novedades Educativas, EUDEBA.

\section{RODRÍGUEZ DELGADO, Rafael}

1994. Teoria de Sistemas y gestión de las organizaciones. Lima, SESGE e IAS.

\section{RODRÍGUEZ VALENCIA, Joaquín}

2003. Introducción a la administración: con enfoque de sistemas. México D. F., Thomson. Cuarta Edición.

ROSENTAL, M. M. y STRAKS, G. M.

1984. Categorías del materialismo dialéctico. México D. F., Grijalbo. 


\section{SÁNCHEZ MARTINES, Eduardo}

2005. Para un planeamiento estratégico de la edtucación: elementos conceptuales y meladologicos. Córdoba (Argentina), Editorial Brujas.

\section{SOLOMÓN, Bárbara}

1999. "Inventarios de estilos de aprendizaje.Universidad de Carolina del Norte". En: Opciones profesionales. Lima, Bruño. Bachillerato Peruano.
UNIVERSIDAD NACIONAL DE SAN ANTONIO ABAD DE CUSCO

2007. Boletín estadístice $\mathcal{N}^{\circ}$ 21. Cusco, Oficina de Planificación Universitaria (Área de Estadística).

2007. Prospecto de admisión 2008. Comisión Permanente de Admisión. Cusco, Editorial Universitaria.

VAN GIGCH, John P.

1990. Teoria ganeral de sistemas México D. F., Trillas Segunda Edición. 